\title{
INVESTIGACIÓN
}

Recibido: 12/03/2021 --- Aceptado: 15/04/2021 --- Publicado: 24/05/2021

\section{COMPORTAMIENTO DE COMPRA Y MARKETING DE LOS SENTIDOS: UN ANÁLISIS DE SU INFLUENCIA EN LOS COMPRADORES DE MODA}

\section{Shopping behaviour and sensory marketing: an analysis of their influence on fashion shoppers}

Pedro Pablo Marín Dueñas': Universidad de Cádiz. España. pablo.marin@uca.es

Diego Gómez Carmona: Universidad de Cádiz. España. diego.gomezcarmona@uca.es

\section{Como citar el artículo:}

Marín Dueñas, P. P. y Gómez Carmona, D. (2021). Comportamiento de compra y marketing de los sentidos: un análisis de su influencia en los compradores de moda. Vivat Academia. Revista de Comunicación, 154, 459-479. http://doi.org/10.15178/va.2021.154.e1366

\section{RESUMEN}

Frente a consumidores cada vez mejor informados y más exigentes, que también buscan que su compra se convierta en una experiencia y a la creciente importancia de los portales de compra online frente al retail físico, se hace necesario el desarrollo de estrategias en el punto de venta para atraer a los compradores y sigan acudiendo a las mismas, no sólo a realizar una compra, sino a pasar tiempo en la tienda. $Y$, en este sentido, una de las grandes diferencias (por no decir el punto fuerte) entre la tienda física y la online es la experiencia física de poder tocar y sentir el producto. La tienda no debe ser entendida solo como un punto de venta, sino que se debe configurar como un espacio donde vivir experiencias. $Y$ es aquí, integrada dentro de la estrategia de trade marketing, donde cobra especial relevancia el marketing de los sentidos. Partiendo de esta premisa, la presente investigación se centra en el estudio del marketing sensorial utilizado por las tiendas de moda del grupo Inditex y, más concretamente, la influencia que el uso de dichas técnicas tiene en el comportamiento de compra de los consumidores y en cómo estos perciben la generación de experiencias. Para ello se aplicará la metodología cuantitativa de la encuesta a una

\footnotetext{
1 Pedro Pablo Marín Dueñas: Doctor por la Universidad de Cádiz. Profesor Ayudante Doctor en el Departamento de Marketing y Comunicación de la Universidad de Cádiz. Coordinador de Grado en Marketing e Investigación de Mercados de la Universidad de Cádiz
} 
Marín Dueñas, P. P. y Gómez Carmona, D.

Comportamiento de compra y marketing de los sentidos: un análisis de su influencia en los compradores de moda

muestra de la población cuyas respuestas se estudian a través del software estadístico SPSS. De los resultados se desprende que si bien el uso de estas técnicas tiene una influencia positiva en los consumidores desde el punto de vista de la experiencia de compra, realmente no les influyen en la decisión de compra final.

PALABRAS CLAVE: Marketing sensorial - Neuromarketing - Retail marketing Trade marketing - Merchandising - Moda - Punto de venta - Influencia Comportamiento de compra

\section{ABSTRACT}

Faced with increasingly better informed and more demanding consumers, who are also looking for their purchase to become an experience, and the growing importance of online shopping portals compared to physical retail, it is necessary to develop strategies at the point of sale to attract shoppers and keep them coming back, not only to make a purchase but also to spend time in the shop. In this sense, one of the big differences (if not the strongest point) between the physical shop and the online store is the physical experience of being able to touch and feel the product. The shop should not only be understood as a point of sale but should also be configured as space where to live experiences. Furthermore, it is here, integrated within the trade marketing strategy, where the senses' marketing takes on special relevance. Based on this premise, this research focuses on the study of sensory marketing used by the Inditex group's fashion shops and, more specifically, the influence that the use of these techniques has on the purchasing behaviour of consumers and how they perceive the generation of experiences. For this purpose, the survey's quantitative methodology will be applied to a sample of the population whose responses are studied using SPSS statistical software. The results show that, although the use of these techniques has a positive influence on consumers from the point of view of the shopping experience, they do not influence them in the final purchase decision.

KEYWORDS: Sensory marketing - Neuromarketing - Retail marketing - Trade marketing - Merchandising - Fashion - Point of sale - Influence - buying behaviour

Agradecimiento: Nuestro agradecimiento al grupo de investigación SEJ-482 Innovación Social en Marketing de la Universidad de Cádiz.

\section{INTRODUCCIÓN}

En una época en la que la compra online está ganando cada vez mayor peso frente al retail tradicional provocando menores ventas en el comercio offline y una cantidad cada vez menor de compradores en tiendas físicas (Emarketer Retail, 2018) se hace imprescindible para éstas convertir el punto de venta en un lugar donde el consumidor se sienta a gusto y pueda disfrutar de la experiencia de compra. Los expertos de la industria minorista sugieren un cambio en el retail para evitar su desaparición y conocer de primera mano los cambios en los hábitos de compra y las 
tendencias actuales de consumo (Internet Retailer, 2018). Frente al denominado modelo brick and click (Gómez y Suárez, 2012) en el que los consumidores compran de manera online a través de un clic de ratón o de las pantallas de sus móviles y tablets, los gestores de los establecimientos físicos deben desarrollar nuevas estrategias de venta que atraigan a los compradores al local, volviendo al modelo brick and mortar o venta tradicional en el que los consumidores acuden al establecimiento a comprar sus productos (Gómez y Suárez, 2012).

Pero el problema para las tiendas físicas no sólo está en el negocio online sino que además tienen que desarrollar su actividad en un mercado sobresaturado de productos competitivos de difícil diferenciación y en el que los consumidores además de estar cada vez mejor informados y ser más exigentes también buscan que su compra se convierta en una experiencia. Tal y como apunta el Informe Consumer Insights Survey 2018 de PWC, el consumidor español acude a las tiendas físicas buscando no solo el producto sino que espera una experiencia de compra sensorial y social. Es decir, la compra se ha convertido en una búsqueda de experiencias envueltas en sensaciones, sentimientos, percepciones, exclusividad, personalización y efectividad (Medina y Quispe, 2019) lo que pone de manifiesto la necesidad de adaptar el marketing a la nueva realidad.

El marketing del siglo XXI debe evolucionar, adaptarse a las nuevas demandas y dejar atrás las estrategias y tácticas orientadas en la venta y promoción. Resulta fundamental para el comercio minorista crear experiencias de compra, conectar con los clientes en un plano emocional y fomentar mejores relaciones

En este sentido, la aplicación de la denominada estrategia de merchandising, definida por Zorrilla (2000) como un conjunto de técnicas que favorecen el acto de compra a partir de la adecuada presentación tanto de los productos como de su entorno, así como la gestión de forma rentable del espacio de la tienda debe dar un paso más y evolucionar en su desarrollo hacia el denominado marketing experiencial que tiene como objetivo crear experiencias de compra a través de las que el consumidor viva momentos diferentes y únicos durante el tiempo que pase en la tienda, evocando reacciones emocionales para crear mejores relaciones con el cliente. Así, para Segura y Sabaté (2008) la elección final del consumidor está determinada por las emociones que le despierte el proceso de compra. Según Schmitt (2007), el marketing ha dado un nuevo giro y "el cliente ya no elige un producto o servicio solo por la ecuación costebeneficio, sino por la vivencia que ofrece antes de la compra y durante su consumo" (p. 8).

Vinculado a este marketing experiencial se encuentra una herramienta que utiliza los estímulos que las personas perciben a través de sus sentidos (vista, tacto, gusto, oído y olfato) para mejorar la experiencia de compra e influir en el comportamiento de los consumidores intentando establecer nuevos vínculos con ellos mediante los sentidos (Castanyol, 2014). Es el denominado marketing sensorial, técnica de comercialización que hace referencia a la interacción empresa-consumidor prestando atención y estimulando todos los sentidos (vista, oído, olfato, tacto y gusto). El 
Marín Dueñas, P. P. y Gómez Carmona, D.

Comportamiento de compra y marketing de los sentidos: un análisis de su influencia en los compradores de moda

marketing de los sentidos permite influir en las percepción, juicios y comportamientos de los consumidores creando un ambiente agradable que incremente el tiempo de compra en el punto de venta (Berčík et al., 2020; BellidoPérez y López-Cortés, 2019; Bilek, Vietoris e Ilko, 2016; Tauferova et al., 2015). Esta técnica supone una ventaja de los detallistas frente al comercio online. Los consumidores pueden experimentar a través de sus sentidos la experiencia en tiendas físicas especialmente en aquellos productos que requieren tocar, sentir y probar previamente a la decisión de compra (Kim et al., 2020). Específicamente, aquellos minoristas que se dedican al retail de moda tienen otra ventaja, dado que a diferencia de otros bienes que no requieren evaluación física antes de su adquisición, la ropa debe verse, tocarse y generar una experiencia previa antes de decidir su compra (Kim et al , 2020). De hecho, esta carencia de experiencia en la compra online de ropa provoca una tasa de devoluciones superior al 40\% (CNBC, 2016).

Por tanto, para conseguir este cambio, será necesario aplicar técnicas sensoriales dentro del comercio minorista físico (Helmefalk y Berndt, 2018) y, de hecho, el marketing de los sentidos ha adquirido en los últimos tiempos una gran trascendencia en el desarrollo de estrategias de atracción del consumidor y como generadora de experiencias de compra puesto que es una manera de conectar con los clientes, despertando en ellos una serie de emociones que tienen mucha más fuerza que los propios aspectos tangibles derivados del producto que se va a adquirir. En definitiva, tal y como apuntan autores como Hultén (2011) o Kumar (2014) estimular los sentidos de los consumidores como elemento diferenciador impactará de manera directa en la fidelidad, recuerdo y comportamiento del consumidor.

Desde la perspectiva académica, Ortegón y Gómez (2016) consideran que si bien la investigación en este campo se ha incrementado en las últimas décadas (Schmitt, 1999; Hultén, 2011; Gómez et al., 2014), el estudio de los sentidos y su aplicación en el marketing es un campo de estudio relativamente reciente (Krishna, 2009), por lo que se hace necesario seguir profundizando en el mismo a partir del desarrollo de la investigación académica. Fruto de esta perspectiva y del interés que despierta este campo de estudio, por la diferenciación que puede suponer aplicar esta estrategia para el retail de moda, surge este trabajo que busca no sólo profundizar en los conceptos teóricos vinculados al marketing sensorial sino, también, analizar la influencia que aplicar estas técnicas en el punto de venta tiene en los consumidores de moda.

\subsection{Conceptualizando el marketing de los sentidos}

Charles, Newman y Wright (2016) definen el marketing de los sentidos como la utilización de estímulos y elementos que los clientes perciben a través de los sentidos

(olfato, vista, oído, tacto y gusto). Para Krishna, Lee y Schwarz (2017) es la combinación de la experiencia sensoriomotriz y la cognición del cliente, con resultados inmersos en el pensamiento, sentimiento y comportamiento de éste. En palabras de Jiménez, Bellido y López (2019, p. 123) "el marketing sensorial en el punto de venta lo entenderemos como aquel que se dirige a los cinco sentidos

Vivat Academia. Revista de Comunicación. 2021, n 154, 459-479 
Marín Dueñas, P. P. y Gómez Carmona, D.

Comportamiento de compra y marketing de los sentidos: un análisis de su influencia en los compradores de moda

buscando la estimulación de estos para intentar crear un ambiente agradable, de forma que el cliente incremente el tiempo de compra en el establecimiento". Krishna (2009, p. 2) lo resume como "el marketing que involucra los sentidos de los consumidores y afecta su comportamiento". Y todo ello con el fin de proporcionar placer estético, emoción, belleza y satisfacción (Schmitt, 2006).

Sea como fuere, Lindstrom (2005) considera que el objetivo del marketing sensorial es el de conectar al consumidor y a la marca a nivel emocional mediante la interacción, durante el proceso de compra, de los cinco sentidos humanos buscando que lo emocional prevalezca sobre lo racional. Estimulando los sentidos se podrá influenciar directamente en la percepción de los consumidores y en su proceso de compra (Megía, 2016).

Esta estrategia que pone en valor la capacidad de los cinco sentidos de los seres humanos para influir en sus decisiones de compra debe implementarse de manera conjunta creando lo que se denomina una experiencia sensorial (Hulten, Broweus y Van Dijk, 2008). Es por esto que es vital el entendimiento de los sentidos para que al ser estimulados en el establecimiento, ayuden a conseguir el objetivo de la venta.

\subsubsection{Olfato}

La asociación de un olor con un establecimiento o un producto puede ser tan intensa que pasa a convertirse en parte de la identidad de la misma marca (Manzano, Gavilán, Avello, Abril y Serra, 2002), estimulando al cliente durante el proceso de compra y despertando su deseo. El olfato es el sentido que genera mayor nivel de recuerdo y evocación (Gómez y García, 2012) y es que conecta de manera directa con las estructuras límbicas del cerebro que son las responsables de nuestras emociones y conductas (González y Pallarés, 2019, Fiore, Niehm y Lorenz, 2008; Goldkuhl y Styfven, 2007). Al oler, por tanto, el sentimiento que se genera es inmediato, no hay influencia de los pensamientos. Con el sentido del olfato, nuestro cerebro actúa antes de pensar, a diferencia de lo que ocurre con los demás sentidos (Manzano et al., 2012).

Surge así, por tanto, entre las herramientas de marketing sensorial el denominado marketing aromático, un factor de gran influencia en el comportamiento de compra de los consumidores. Los seres humanos tenemos la capacidad de rememorar el 35\% de lo que olemos y distinguir aproximadamente entre 10000 olores distintos, así como asociar determinados olores a situaciones puntuales. Y no sólo eso, una investigación actual afirma que el $75 \%$ de nuestras emociones diarias están relacionadas con los aromas (Berčík et al., 2020). Estas emociones influyen en el recuerdo generado por el estímulo y permite recordar algo que olemos mejor que algo que vemos, oímos o tocamos (Vlahos, 2007). En este sentido, las empresas pueden facilitar el reconocimiento del producto vinculando su identidad corporativa con un aroma y así diferenciar la marca de sus competidores. Además, un olor agradable en el punto de venta mejora un $44 \%$ el tiempo de permanencia del consumidor en tienda (Conick, 2017) y, por ende, el ticket medio de su compra 
Marín Dueñas, P. P. y Gómez Carmona, D.

Comportamiento de compra y marketing de los sentidos: un análisis de su influencia en los compradores de moda

podría aumentar (Spectrio, 2020). Es el caso, por ejemplo, de los aromes dulces y cálidos, como el de la canela o la vainilla, que se usan en las tiendas de moda. La correcta aplicación del aroma contribuye a mejorar la percepción que tienen los clientes del establecimiento, sus productos y servicios. También se ha demostrado que el marketing aromático aumenta las visitas al punto de venta (ScentAir, 2020).

Además, la percepción de un olor puede variar en función de la persona y estar influido por aspectos individuales como el género o la edad. Según Hirsch y Gay (1991) según el sexo de la persona se tienen diferentes reacciones ante el mismo olor siendo los hombres, por ejemplo, menos sensibles a los olores que las mujeres. Así un hombre puede pasar más tiempo ante un estante con olor picante y ser menos susceptible al olor de champú que las mujeres. Desde el punto de vista de la edad, el sentido del olfato se va debilitando conforme las personas se van haciendo mayores (Barrios, 2012, p. 77).

Iannini (2010) diferencia entre tres tipos de aromas, los identificativos, denominados odotipos o logos olfativos a través de los que una marca crea su identidad olfativa que le permite ser reconocida por los consumidores como son conocidas por su identidad visual. El odotipo es, por tanto, el olor de una marca que transmite sus valores e intereses a sus públicos a través de él. Habla Iannini, en segundo término, de los aromas asociativos encargados de estimular las ventas en el punto de venta a partir de aromas creados adhoc. Finalmente este autor identifica lo que él denomina aromas reproductivos, y que reproducen con exactitud el olor de un producto o lo potencian para fomentar su consumo.

Centrados en la aplicación del marketing olfativo en el punto de venta, Manzano et al. (2012) diferencian tres: la generación de tráfico (un aroma bien elegido y aplicado en la entrada o en el exterior, cerca del acceso al establecimiento, puede atraer clientes al negocio); la ambientación (aromatizar estratégicamente el local para mejorar la respuesta del cliente, buscando un efecto de relajación y bienestar que facilite la circulación del cliente y le haga olvidar el paso del tiempo); la señalización (utilizar los olores como advertencia, aviso o reclamo -por ejemplo de un nuevo producto o destacar una sección concreta-. Busca, en definitiva, dirigir, atraer, orientar o indicar).

Entre las ventajas que se derivan de aplicar este marketing olfativo o aromático está el hecho de que crea experiencias para el consumidor llegando incluso a modificar conductas y posicionando la marca en el top of mind del consumidor ya que el olfato tiene memoria. Asocia valores a la marca que se relacionan con el aroma y vincula al consumidor con el mundo de la misma, pudiendo llegar a retener a los consumidores en un establecimiento comercial y fidelizando a los mismos.

\subsubsection{Tacto}


Marín Dueñas, P. P. y Gómez Carmona, D.

Comportamiento de compra y marketing de los sentidos: un análisis de su influencia en los compradores de moda

Las sensaciones de la piel alteran los estados de ánimo, es por eso, que se considera al sentido del tacto importante para las interacciones comerciales y es que este sentido permite interactuar con los productos, acercando éstos a los clientes. Si se compara con los demás sentidos el tacto es el único que permite el contacto físico directo con el producto, jugando un papel clave en la decisión de compra. Así, a partir de los distintos receptores táctiles que están ubicados en nuestra piel se produce la percepción háptica, y "toda esta información háptica es transmitida al cerebro, quien busca su asociación con experiencias archivadas en él o la procesa como nueva, para así generar la respuesta más adecuada que se traduzca en un sentido o una acción" (Manzano et al., 2012 p. 178).

Derivado de esta percepción háptica surge otra de las herramientas de marketing sensorial que pueden emplear los detallistas de ropa en sus establecimientos: el marketing háptico, un aspecto fundamental para la industria de la moda. Concretamente, el consumidor de moda necesita tocar y sentir la ropa antes de comprarla. Los informes de la industria textil y de confección indican que aproximadamente el $85 \%$ de los consumidores eligen comprar en tiendas físicas precisamente porque permiten apreciar las características como la textura o la suavidad (TimeTrade, 2017). Por ejemplo, tocar un suéter puede afectar las percepciones de textura, dureza y peso (Klatzky y Lederman, 1993; Klatzky y Lederman, 1992). Concretamente, el atributo suavidad es el más importante para evaluar la calidad del producto (Monitor TM 2017; Chang et al., 2015) y es imposible apreciarlo mediante la vista (Spence et al., 2014). Para consumidores que experimentan las percepciones de suavidad mediante el tacto con su propio cuerpo, la percepción de la textura permitirá evaluar el producto y determinar la decisión de compra (Workman, 2010; Childers y Peck, 2010). Por tanto, mejorar las percepciones de suavidad háptica en los productos podría mejorar la experiencia del consumidor en el establecimiento.

\subsubsection{Vista}

La vista es el sentido más poderoso pues permite captar la información del exterior $\mathrm{y}$, por tanto, es el más utilizado desde el punto de vista del marketing y la comunicación (González y Pallarés, 2019). A través del sentido de la vista una persona percibe el mundo que le rodea. En su día a día un consumidor recibe miles y miles de estímulos visuales (anuncios, carteles, envases, señalizaciones) que le ofrecen información y que le predisponen a pensar o actuar de una determinada manera (Manzano et al., 2012). Dada su importancia, de los cinco sentidos, el de la vista es el más conocido y explotado en una estrategia de marketing sensorial, predominando sobre las demás modalidades sensoriales (Biswas et al., 2019; Biswas et al., 2014). Es el denominado marketing visual, definido por Manzano et al. como "la utilización estratégica que las compañías realizan de estímulos, signos y símbolos comerciales y no comerciales para comunicar mensajes a sus consumidores" (2012, p. 95). 
Marín Dueñas, P. P. y Gómez Carmona, D.

Comportamiento de compra y marketing de los sentidos: un análisis de su influencia en los compradores de moda

Manzano et al. (20012) realiza una clasificación del impacto sobre el consumidor que el marketing visual tiene en el punto de venta (Figura 1).

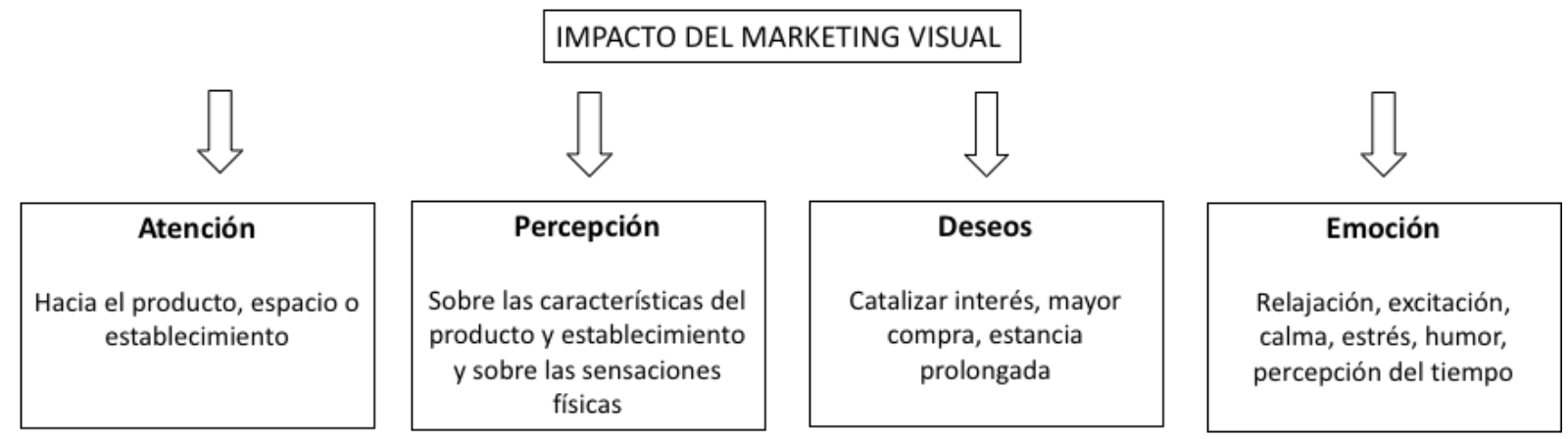

Figura 1: Clasificación de los efectos del marketing visual en el punto de venta

Fuente: Comunicar con los sentidos en el punto de venta (Manzano et al., 2012)

Para su análisis comportamental se ha utilizado tradicionalmente el modelo Estímulo Organismo Respuesta (para un ejemplo, Koo y Kim, 2013). En este modelo se describe explícitamente hasta qué punto las señales visuales del punto de venta tienen un impacto en la percepción, la emoción, y el comportamiento de compra del consumidor (Morone et al., 2018; Kühn y Petzer, 2018).

Entre los factores que se han analizado sobre marketing visual en el punto de venta se encuentran:

1. El color (Bagchi y Cheema 2013; Mehta y Zhu 2009), elemento determinante para influir en el estado afectivo, cognitivo y comportamental de los clientes que entran en el establecimiento (Díez de Castro et al., 2006). Aquí se podría incluir la iluminación, factor clave en cualquier establecimiento comercial. Dota de mayor visibilidad a los productos, permite crear distintos ambientes e influye en como el consumidor percibe la mercancía y en la capacidad de atracción del propio local (Gómez y García, 2012).

2. La orientación de la cartelería en el establecimiento (Romero y Biswas, 2016)

3. La forma de disponer el mobiliario (Jiang et al., 2016). Estamos hablando del diseño interior, referido a la decoración, accesibilidad, la disposición del mobiliario, materiales y estilo usado, entre otros y que influyen en el tránsito del consumidor por la tienda y en el tiempo que permanece en ella (Gómez y García, 2012)

4. El dinamismo en la ubicación y rotación de productos (Roggeveen et al., 2015; Cian, Krishna y Elder, 2015).

5. El diseño exterior (que incluye elementos como el escaparate, la fachada, las puertas de acceso o los rótulos y la entrada) se configura también como un factor de atracción clave del establecimiento. Kotler et al. (2008) consideran que el viandante se genera unas expectativas sobre el establecimiento cuando ve su fachada y su escaparate que, por otro lado, deberían cumplirse en el interior. Este diseño exterior debería atraer al cliente captando su atención y motivándole a acceder al establecimiento (Díez de Castro et al., 2006). 
En el caso particular del sector moda Rodríguez (2018) sugiere desarrollar estrategias de marketing visual creando emociones en el punto de venta. Esta autora afirma que así el establecimiento alcanzará un reconocimiento de marca efectivo y coherente que mejorará el posicionamiento y reducirá las decisiones de compra. Para analizar las emociones generadas por distintas distribuciones (parrilla vs. libre) de un establecimiento de moda Jang et al. (2018) estudiaron el efecto de la complejidad visual. Los resultados demuestran que la complejidad visual de la tienda influye negativamente sobre el placer cuando la implicación de los consumidores con la moda es bajo, y que este efecto disminuye en consumidores con alta implicación. Además, la complejidad visual se relaciona con una mayor excitación emocional. Junto a los procesos emocionales, una investigación analizó la percepción de los consumidores cuando se encuentran en un establecimiento abarrotado, concluyendo que la aglomeración puede desplazar las preferencias de los consumidores hacia productos y marcas que provocan mayores respuestas afectivas (Aydinli, 2020).

\subsubsection{Oído}

Junto a las herramientas sensoriales anteriores, los expertos en marketing pueden manipular los mensajes de comunicación dirigidos a los consumidores a través de la megafonía y la música, como un elemento atmosférico en la tienda (ReynoldsMcIlnay y Morrin, 2019; Biswas, Lund y Szocs, 2019; Roschk, Loureiro y Breitsohl 2017). “La música, la voz humana y los sonidos en general aplicados al marketing no deberían ser fruto del azar, sino una herramienta más de la estrategia del negocio (Manzano et al., 2012, p. 117).

Las comunicaciones dirigidas al consumidor por lo general suelen aumentar la confianza de los clientes en los establecimientos minoristas (Guenzi, Johnson y Castaldo 2009). La música que los consumidores escuchan puede cambiar sus percepciones sobre el establecimiento y los productos que se comercializan. Es posible actuar sobre algunos elementos musicales como a) la armonía o el timbre (Bruner 1990), b) la frecuencia que a menudo se percibe como el tono (Lowe et al., 2018) y c) la amplitud que se mide en decibelios y se percibe como el volumen o la suavidad del sonido (Krishna, 2013). El tratamiento de estos elementos auditivos es de los más variado, si bien algunos establecimientos utilizan la misma música para todo el punto de venta otros prefieren zonificar la superficie de venta con música distinta dependiendo del tipo de producto (Cleaver, 2016; Haugh, 2016).

Los resultados respecto al comportamiento del cliente indican efectos divergentes sobre la presencia de música en el punto de venta (Baumann y Gayer, 2017). Si bien algunos autores informan de una presencia 15 minutos superior del consumidor en la tienda (Andersson et al., 2012; Sullivan, 2002) otros trabajos no identifican diferencias significativas entre utilizar o no música (Wilson, 2003; North et al., 2003).

Algunos autores sugieren que poner música en la tienda hace que los clientes se sientan mejor, lo que los lleva a gastar más dinero (Baumann y Gayer, 2017), aunque 
Marín Dueñas, P. P. y Gómez Carmona, D.

Comportamiento de compra y marketing de los sentidos: un análisis de su influencia en los compradores de moda

esta afirmación no es apoyada por toda la comunidad científica (North et al., 2015; Milliman, 1982). En resumen, investigaciones anteriores muestran efectos mixtos provocados por diferentes razones como el diseño de la música, el tipo de establecimiento o la relación entre la música, el consumidor y la tienda (Baumann y Gayer, 2017).

\subsubsection{Gusto}

Desde el punto de vista del sector detallista la experiencia sensorial derivada del sentido del gusto puede ser una herramienta comercial eficaz para conseguir atraer a los consumidores, incrementar las ventas y conseguir una imagen diferencial, reforzando la vinculación de los consumidores con la enseña y sus establecimientos (Manzano et al., 2012) si bien esto es especialmente relevante en aquellas organizaciones que distribuyen productos de alimentación. En el caso concreto de este estudio, que analiza el sector de la distribución de moda, apenas tiene influencia por lo que se puede afirmar que se trata de una herramienta de marketing sensorial que no implementan estas marcas, salvo casos excepcionales como alguna bebida o alimento (café, champagne, bombones...) que puedan ofrecer algunas tiendas de lujo o algún tipo de caramelo que puedan ofrecer otros negocios.

\section{OBJETIVOS}

El objeto de estudio de la presente investigación es el sector retail de moda y, más concretamente, la influencia que la aplicación de las técnicas de marketing sensorial por parte de los establecimientos de moda del grupo Inditex (Zara y Stradivarius) tiene en el comportamiento de los consumidores.

Derivado de dicho objeto de estudio, el objetivo general del estudio es analizar el efecto del marketing sensorial en el comportamiento de compra de los consumidores de moda. Específicamente se analizará:

1. La importancia que las técnicas de marketing sensorial tienen para los consumidores

2. La percepción que estos consumidores tienen frente a las distintas técnicas de marketing sensorial implementadas en la tienda

3. El impacto, desde el punto de vista del estado de ánimo y los sentimientos, que las acciones de marketing sensorial tienen en el consumidor

4. El motivo principal por el que finalmente han realizado una compra

\section{METODOLOGÍA}

La herramienta metodológica que se ha usado para dar respuesta a los objetivos que se han marcado es la técnica cuantitativa de la encuesta, definida por López y Fachelli (2016, p. 8) como "una técnica de recogida de datos a través de la interrogación de los sujetos cuya finalidad es la de obtener de manera sistemática medidas sobre los conceptos que se derivan de una problemática de investigación previamente construida" y que, en el caso concreto de esta investigación, permite la 
Marín Dueñas, P. P. y Gómez Carmona, D.

Comportamiento de compra y marketing de los sentidos: un análisis de su influencia en los compradores de moda

medición científica de este fenómeno social a través de la recolección de los datos de una manera estandarizada a partir de una batería de preguntas, que siempre son las mismas para todos los encuestados (Cea, 2012).

Para su puesta en práctica, se ha elaborado un cuestionario ad-hoc a partir de las investigaciones de Manzano et al. (2012), Morgan (2016) y Hervas, Campo y Revilla (2013).

Más específicamente, se definieron las siguientes variables de análisis:

1. Nivel de importancia

2. Efecto de la técnica en el consumidor

3. Impacto en el consumidor

4. Motivación de compra del consumidor

Para recabar las respuestas se aplicó un muestreo no probabilístico (de conveniencia por accesibilidad). Concretamente se seleccionaban a aquellos clientes de más de 16 años que salían de los puntos de venta objeto del estudio (las tiendas Zara y Stradivarius situadas en el Centro Comercial Área Sur, en Jerez de la Frontera (Cádiz)) y que tenían que cumplir el condicionante de haber realizado una compra. El total de personas que han respondido a dicha encuesta y que se configuran como muestra final de esta investigación es de 140 personas -total de personas que, el día elegido para visitar el establecimiento, realizaron una compra en el mismo y aceptaron participar en el estudio- (70 para Stradivarius y 70 para Zara) y que se distribuyen de la siguiente manera: un $74 \%$ de mujeres por un $26 \%$ de hombres. En cuanto al tramo etario, el 73\% tienen entre 16 y 30 años, el 17\% entre 31 y 45 años, un $7 \%$ se sitúa entre los 46 y los 60 años y el 3\% restante tenía más de 60 años. Hay que señalar que las diferencias tanto por género como por edad entre ambos establecimientos no son significativas.

\section{RESULTADOS}

En relación al grado de importancia que los consumidores conceden a la aplicación de técnicas de marketing visual, en general coinciden en dar mucha importancia a aquellas que tienen que ver con el producto en sí. Esto es, tanto la distribución del mobiliario en la tienda y a la accesibilidad a la ropa como la calidad y diseño de los productos son muy importantes para los clientes. Lo mismo ocurre con el orden y limpieza del establecimiento y el trato personal. Por el contrario, las relacionadas con el marketing auditivo no tienen importancia para ellos. En cuanto al resto de variables, tanto la iluminación como el aroma son consideradas como importantes durante el proceso de compra.

Si se profundiza en la valoración que hacen de la aplicación de estas técnicas en la propia tienda y se relaciona con ese nivel de importancia los resultados son positivos. Si se analizan las variables consideradas como muy importantes (distribución del mobiliario, calidad y diseño, accesibilidad a las prendas, orden y limpieza y trato personal) el efecto que producen en el consumidor de Zara y Stradivarius es 
Marín Dueñas, P. P. y Gómez Carmona, D.

Comportamiento de compra y marketing de los sentidos: un análisis de su influencia en los compradores de moda

satisfactorio o muy satisfactorio. En el caso del marketing auditivo, que resulta poco importante para los compradores, tiene un efecto neutro en Zara si bien en Stradivarius es muy satisfactorio para el indicador género musical y satisfactorio para el volumen. En cuanto al resto de variables estudiadas, el aroma, si bien es considerado como importante para el cliente de Zara, éste no provoca ningún efecto en él, siendo satisfactorio en el caso del consumidor de Stradivarius. Finalmente, en este análisis, destaca que la iluminación de los probadores de Stradivarius no es nada satisfactoria, si bien en el resto de zonas, en ambos establecimientos, ésta es adecuada.

Tabla 1: Grado de importancia y efecto sobre el consumidor del marketing sensorial

\begin{tabular}{|c|c|c|c|c|}
\hline \multirow{2}{*}{$\begin{array}{l}\text { Tipo de } \\
\text { técnica }\end{array}$} & \multirow{2}{*}{$\begin{array}{c}\text { Aspectos que } \\
\text { se evalúan }\end{array}$} & \multirow{2}{*}{$\begin{array}{l}\text { Grado de } \\
\text { importancia }\end{array}$} & \multicolumn{2}{|c|}{$\begin{array}{l}\text { Efecto predominante sobre el } \\
\text { consumidor (en función del } \\
\text { establecimiento) }\end{array}$} \\
\hline & & & ZARA & STRADIVARIUS \\
\hline \multirow{7}{*}{$\begin{array}{l}\text { Marketing } \\
\text { visual }\end{array}$} & $\begin{array}{l}\text { Color presente } \\
\text { en la tienda }\end{array}$ & Poco importante & Satisfactorio & Neutro \\
\hline & $\begin{array}{l}\text { Iluminación de } \\
\text { estanterías y } \\
\text { expositores }\end{array}$ & Importante & Satisfactorio & Satisfactorio \\
\hline & $\begin{array}{l}\text { Iluminación } \\
\text { del escaparate }\end{array}$ & Importante & Satisfactorio & Satisfactorio \\
\hline & $\begin{array}{l}\text { Iluminación de } \\
\text { los probadores }\end{array}$ & Importante & Satisfactorio & Nada satisfactorio \\
\hline & $\begin{array}{l}\text { Decoración } \\
\text { interior }\end{array}$ & Importante & Satisfactorio & Satisfactorio \\
\hline & $\begin{array}{l}\text { Distribución } \\
\text { general del } \\
\text { mobiliario }\end{array}$ & Muy importante & Satisfactorio & Muy satisfactorio \\
\hline & $\begin{array}{l}\text { Acceso a las } \\
\text { prendas }\end{array}$ & Muy importante & $\begin{array}{c}\text { Muy } \\
\text { satisfactorio }\end{array}$ & Muy satisfactorio \\
\hline \multirow{3}{*}{$\begin{array}{l}\text { Marketing } \\
\text { auditivo }\end{array}$} & $\begin{array}{l}\text { Género } \\
\text { musical }\end{array}$ & Poco importante & Neutro & Muy satisfactorio \\
\hline & Volumen & Poco importante & Neutro & Satisfactorio \\
\hline & Tempo y ritmo & Nada importante & Neutro & Neutro \\
\hline $\begin{array}{l}\text { Marketing } \\
\text { olfativo }\end{array}$ & Aroma & Importante & Neutro & Satisfactorio \\
\hline \multirow{3}{*}{$\begin{array}{l}\text { Marketing } \\
\text { táctil }\end{array}$} & $\begin{array}{l}\text { Calidad y } \\
\text { diseño de los } \\
\text { productos }\end{array}$ & Muy importante & Satisfactorio & Satisfactorio \\
\hline & $\begin{array}{l}\text { Orden y } \\
\text { limpieza }\end{array}$ & Muy importante & $\begin{array}{c}\text { Muy } \\
\text { satisfactorio }\end{array}$ & Satisfactorio \\
\hline & Trato personal & Muy importante & $\begin{array}{c}\text { Muy } \\
\text { satisfactorio }\end{array}$ & Satisfactorio \\
\hline
\end{tabular}

Fuente: Elaboración propia

Una vez analizadas estas cuestiones, se quiso profundizar en el impacto, desde el punto de vista del estado de ánimo y los sentimientos, que estas variables de marketing sensorial habían tenido en el consumidor tras su paso por el 
Marín Dueñas, P. P. y Gómez Carmona, D.

Comportamiento de compra y marketing de los sentidos: un análisis de su influencia en los compradores de moda

establecimiento. En este sentido todas fueron positivas generando en el cliente un estado de satisfacción, tranquilidad y confianza, lo que afectó de manera positiva su comportamiento de compra.

Finalmente, también se indagó sobre cuál fue el motivo principal que llevó al consumidor a realizar una compra, independientemente de que estuviese relacionado o no con el marketing sensorial. Lo que más influenció a la hora de tomar una decisión de compra fue el precio $(50,62 \%)$ y la calidad del producto $(50,62 \%)$ seguidos ya de lejos por las promociones $(27,16 \%)$. Como se puede comprobar, una de los indicadores vinculados al marketing táctil es el principal motivo de la compra si bien éste no depende tanto del propio punto de venta. $\mathrm{Al}$ analizar las variables de marketing sensorial que sí motivaron la compra destacan el orden y la limpieza (39\%), el trato personal $(23,46 \%)$ y la distribución de las prendas $(16 \%)$.

\section{DISCUSIÓN}

Los hallazgos derivados de esta investigación permiten ordenar la importancia de las técnicas de marketing sensorial aplicadas en Zara y Stradivarius descubriendo que para los consumidores de estas tiendas el marketing háptico o tactil es el elemento más importante, seguido del marketing visual, el olfativo y el auditivo. Los datos demuestran que sendos establecimientos están perdiendo la oportunidad de explotar las posibilidades que brinda la comunicación auditiva dentro del establecimiento. Esto se refiere no al uso de música, sino a la posibilidad de enviar mensajes persuasivos a los consumidores que alienten el consumo de un determinado tipo de producto en un momento determinado.

Este trabajo, igualmente, ha demostrado la influencia que las técnicas de marketing sensorial implementadas en Zara y Stradivarius tienen en el comportamiento del consumidor. En general las técnicas de marketing visual empleadas en Zara consiguen que el consumidor esté satisfecho. Los resultados en el caso de Stradivarius vienen a indicar resultados similares, aunque presentan algo más de dispersión. En ambos casos la estrategia de marketing visual en el punto de venta aún tiene margen de mejora ya que en ningún caso las herramientas empleadas dejan al consumidor completamente satisfecho. En el caso del marketing auditivo, los resultados indican que la labor de Stradivarius sobre este sentido es mejor que la realizada en el establecimiento comercial de Zara. Es posible que Zara no esté aplicando esta técnica de marketing sensorial por lo que, considerando el corpus teórico, estaría perdiendo la oportunidad de mejorar la experiencia del consumidor durante el proceso de compra. Es decir, estaría desaprovechando la oportunidad de que el consumidor pase más tiempo en el establecimiento, gaste más dinero y tenga más confianza en el mismo. La estrategia de marketing olfativo seguida por Stradivarius también parece ser más valorada que la empleada por Zara, permitiendo diferenciar la marca de sus competidores, lo que puede producir un mayor recuerdo del producto y de la marca mejorando, además, las visitas a tienda. En el caso del marketing háptico los resultados muestran una preferencia por la

Vivat Academia. Revista de Comunicación. 2021, nº 154, 459-479 
Marín Dueñas, P. P. y Gómez Carmona, D.

Comportamiento de compra y marketing de los sentidos: un análisis de su influencia en los compradores de moda

estrategia de Zara frente a Stradivarius. Es posible que los consumidores perciban una mayor calidad en las prendas de Zara derivada de su textura, materiales usados y diseño.

Al estudiar el estado de ánimo y los sentimientos provocados por las acciones de marketing sensorial se ha encontrado que la visita a la tienda, en general, provoca satisfacción, tranquilidad y confianza entre los consumidores. Aspectos que resultan fundamentales para reducir la disonancia postcompra y mejorar la intención de revisita al establecimiento.

Por último, también se halló que los principales motivos para comprar en estos establecimientos son el precio (variable independiente del marketing sensorial) y la calidad del producto (variable vinculada a la estrategia de marketing háptico) y que, y esto es lo relevante, el peso de ambos factores coincide en orden de importancia, lo que refleja que el consumidor que compra en estos establecimientos no está únicamente preocupado por el precio. También es importante destacar que el resto de variables relativas al marketing de los sentidos no tienen mucho peso a la hora de influir en la decisión de compra.

\section{CONCLUSIONES}

Las principales conclusiones extraídas de la literatura parecen mostrar que cada vez más los investigadores y las empresas son conscientes de la importancia que tiene el marketing sensorial. Esto se manifiesta en el creciente número de investigaciones vinculadas al marketing sensorial que recientemente se están realizando. Concretamente los trabajos previos se suelen centrar en uno o dos sentidos al realizar sus investigaciones. También es usual encontrar literatura que analiza el aspecto visual utilizando modernas metodologías como el eye-tracking o el análisis facial y diseños experimentales exploratorios que emplean olores o incluso sabores, dependiendo del contexto.

En el caso de la presente investigación, a través de la metodología de la encuesta, se ha querido profundizar en el efecto que estimular todos los sentidos -salvo el del gusto- en un establecimiento del sector de la moda tiene en el comportamiento de compra de los consumidores. Y la principal conclusión que se puede derivar del estudio es que aún hay margen para la mejora en la implementación del marketing sensorial. Si bien se están poniendo en práctica aún no logran influir de manera decisiva en las decisiones de los compradores y aunque afectan al estado de ánimo de manera positiva, siguen siendo el precio y el producto, dos de las 4 variables tradicionales del mix de marketing, las que más influyen en dicho comportamiento del consumidor, si bien es cierto que la calidad del producto se vincula a la estrategia de marketing háptico.

En definitiva, aún queda un largo camino por recorrer en este proceso que busca crear en las tiendas físicas del sector moda experiencias de compra que generen, como apuntan Medina y Quispe (2019), sensaciones, sentimientos, percepciones, 
Marín Dueñas, P. P. y Gómez Carmona, D.

Comportamiento de compra y marketing de los sentidos: un análisis de su influencia en los compradores de moda

exclusividad y personalización que hagan que el consumidor siga prefiriendo este tipo de compra presencial frente a la compra online.

\section{REFERENCIAS}

Andersson, P. K., Kristensson, P., Wästlund, E. y Gustafsson, A. (2012). Let the music play or not: The influence of background music on consumer behavior. Journal of retailing and consumer services, 19(6), 553-560. https://doi.org/10.1016/j.jretconser.2012.06.010

Aydinli, A., Lamey, L., Millet, K., ter Braak, A. y Vuegen, M. (2020). ¿Cómo modifican los clientes la composición de su cesta cuando perciben que la tienda está abarrotada? Un Estudio Empírico. Revista de venta al por menor.

Bagchi, R. y Cheema, A. (2013). The effect of red background color on willingness-topay: the moderating role of selling mechanism. Journal of Consumer Research, 39(5), 947-960. https:// doi.org/10.1086/666466

Barrios, M. (2012). Marketing de la Experiencia: principales conceptos y características. Palermo Business Review, 7(1), 67-83.

Berčík, J., Mravcová, A., Gálová, J. y Mikláš, M. (2020). The use of consumer neuroscience in aroma marketing of a service company. Potravinarstvo Slovak Journal of Food Sciences, 14, 1200-1210. https:// doi.org/10.5219/1465

Bilek, M., Vietoris, V. y Ilko, V. (2016). Shelf life extension and sensory evaluation of birch tree sap using chemical preservatives. Potravinarstvo Slovak Journal of Food Sciences, 10(1), 499-505. https://doi.org/10.5219/649

Biswas D. y Szocs, C. (2019). The Smell of Healthy Choices: Cross-Modal Sensory Compensation Effects of Ambient Scent on Food Purchases. Journal of Marketing Research, 56(1), 123-141. https:// doi.org/10.1177/0022243718820585

Bruner, G. C. (1990). Music, mood, and marketing. Journal of marketing, 54(4), 94-104.

Castanyol, E. (2014). Marketing sensorial: comunicación a través de los sentidos. COMeIN - Revista de los estudios de ciencias de la información y comunicación, 38. https://doi.org/10.7238/c.n38.1479

Castaño, R. y Pérez, M. E. (2014). A matter of love: consumers' relationships with original brands and their counterfeits. Journal of consumer marketing, 31(6/7), 475482. https:// doi.org/10.1108/JCM-05-2014-0970

Castro, E. C. D. D., Bercebal, F. J. L. y García, A. N. (2006). Merchandising: teoría y práctica. Pirámide. 
Marín Dueñas, P. P. y Gómez Carmona, D.

Comportamiento de compra y marketing de los sentidos: un análisis de su influencia en los compradores de moda

Chang, H. J., Song, J., Yeo, C. y Kim, J. (2015). Exploring factors influencing perceived quality on sportswear fabric. International Textile and Apparel Association Annual Conference Proceedings, 72(1). Iowa State University Digital Press.

Charles, D., Newman, A. y Wright, L. (2016). Enhancing consumer empowerment. European Journal of Marketing, 40(9), 925-935. https://doi.org/10.1108/03090560610680934

Chicaiza, R. P. M. y García, J. Q. (2019). Acercamiento teórico al marketing sensorial: sentidos, experiencias de marcas y modelos. 593 Digital Publisher CEIT, 4(3), 4-16.

Childers, T. L. y Peck, J. (2010). Informational and affective influences of haptics on product evaluation: Is what I say how I feel? En A. Krishna (Ed.), Sensory Marketing: Research on the Sensuality of Products (63-72). Routledge/Taylor \& Francis Group.

Cian, L., Krishna, A. y Elder, R. S. (2015). A sign of things to come: behavioral change through dynamic iconography. Journal of Consumer Research, 41(6), 1426-1446. https://doi.org/10.1086/680673

Conick, H. (2017). The past, present and future of AI in marketing. Marketing News, 51(1), 26-35.

CNBC (2016). A $\$ 260$ billion 'ticking time bomb': the costly business of retail returns. www.cnbc.com/2016/12/16/a-260-billion-ticking-time-bomb-the-costly-business -of-retailreturns.html

Cleaver, E. (2016). How to Help. Consumer Equality: Race and the American Marketplace, 167.

Díez de Castro, E., Landa, F. y Navarro, A. (2006). Merchandising. Teoría y práctica. Ediciones Pirámide.

eMarketer. (2018). Worldwide Retail and Ecommerce Sales: eMarketer's Updated Forecast and New Mcommerce Estimates for 2016-2021.

Goldkuhl, L. y Styvén, M. (2007). Sensing the scent of service success. European Journal of Marketing, 41(11/12), 1297-1305. https://doi.org/10.1108/03090560710821189

Gómez, M. y García, C. (2012). Marketing sensorial, como desarrollar la atmosfera del establecimiento comercial. Distribución y consumo, 24, 30-39.

Gómez, M. y García, C. (2014). The use of sensorial marketing in stores: attracting clients through the senses. En F. Musso y E. Druica (Eds.) Advances in Marketing, Customer Relationship Management, and E-Services. Hershey PA 
Marín Dueñas, P. P. y Gómez Carmona, D.

Comportamiento de compra y marketing de los sentidos: un análisis de su influencia en los compradores de moda

Gonchigjav, B. (2020). Results of neuromarketing study of visual attention and emotions of buyers in retail store environment. Proceedings of the Mongolian Academy of Sciences, 52-64.

González Z. F. y Pallarés, C. (2020): La experiencia Nespresso, el marketing sensorial aplicado al sector del café. Miguel Hernández Communication Journal, 11 (1), 75 96. http://dx.doi.org/10.21134/mhcj.v11i0.325

Guenzi, P., Johnson, M. D. y Castaldo, S. (2009). A comprehensive model of customer trust in two retail stores. Journal of Service Management, 20 (3), 290-316. https://doi.org/10.1108/09564230910964408

Helmefalk, M. y Berndt, A. (2018). Shedding light on the use of single and multisensory cues and their effect on consumer behaviours. International Journal of Retail \& Distribution Management, 46 (2). https://doi.org/10.1108/IJRDM-03-2018$\underline{0057}$

Hirsch, A. R. y Gay, S. E. (1991). The effect of ambient olfactory stimuli on the evaluation of a common consumer product. Chemical Senses, 16(5), 535. https://doi.org/10.5539/ijms.v6n1p155

Hultén, B. (2011). Sensory marketing: the multi-sensory brand-experience concept. $\begin{array}{lllll}\text { European Business } & \text { Review, } & \text { 256-273. }\end{array}$ https://doi.org/10.1108/09555341111130245

Hultén, B., Broweus, N. y Van Dijk, M. (2009). What is sensory marketing? Sensory marketing, (1-23). https://doi.org/10.1057/9780230237049_1

Hultén, B. (2011). Sensory Marketing: The Multi-Sensory Brand-Experience Concept. European Business Review. 23 (3), 56-273.

IAB Spain (2019). Estudio anual de ecommerce. https://iabspain.es/estudio/estudio-anual-de-ecommerce-2019/

Iannini, M. (2010). Marketing olfativo, un valor diferencial. MK: Marketing+ ventas, $253,58-64$.

Internet Retailer (2018). A decade in review: E-commerce sales vs retail sales 20072017.

Jang, J. Y., Baek, E., Yoon, S. Y. y Choo, H. J. (2018). Store design: Visual complexity and consumer responses. International Journal of Design, 12(2), 105-118. 
Marín Dueñas, P. P. y Gómez Carmona, D.

Comportamiento de compra y marketing de los sentidos: un análisis de su influencia en los compradores de moda

Jeong, S. W., Fiore, A. M., Niehm, L. S. y Lorenz, F. O. (2009). The role of experiential value in online shopping. Internet Research. 19 (1), 105-124. https://doi.org/10.1108/10662240910927858

Jiang, Y., Gorn, G. J., Galli, M. y Chattopadhyay, A. (2016). Does your company have the right logo? How and why circular-and angular-logo shapes influence brand attribute judgments. Journal of Consumer Research, 42(5), 709-726. https://doi.org/10.1093/jcr/ucv049

Jiménez, G., Bellido, E. y López-Cortés, A. (2019). Marketing sensorial: el concepto, sus técnicas y su aplicación en el punto de venta. Vivat Academia. Revista de Comunicación, 148, 121-147. http:/ / doi.org/10.15178/va.2019.148.121-147

Kim, J. H., Kim, M., Yoo, J. y Park, M. (2020). Consumer decision-making in a retail store: the role of mental imagery and gender difference. International Journal of Retail \& Distribution Management. 49 (3), 421-445. https://doi.org/10.1108/IJRDM$\underline{10-2019-0353}$

Klatzky, R. L. y Lederman, S. J. (1992). Stages of manual exploration in haptic object identification. Perception \& Psychophysics, 52(6), 661-670.

Klatzky, R. L., Lederman, S. J. y Matula, D. E. (1993). Haptic exploration in the presence of vision. Journal of Experimental Psychology: Human Perception and Performance, 19(4), 726.

Koo, W. y Kim, Y. K. (2013). Impacts of store environmental cues on store love and loyalty: single-brand apparel retailers. Journal of International Consumer Marketing, 25(2), 94-106. https:/ / doi.org/10.1080/08961530.2013.759044

Kotler, P. y Lee, N. (2008). Social marketing: Influencing behaviors for good. Sage.

Krishna, A. (2009). Cognition and sensory perception: the impact of input from sensory modalities in imagery, memory, information processing, and sensory perception. Advances in Consumer Research, 36, 127-130.

Krishna, A. (2013). Customer Sense. Palgrave Macmillan.

Krishna, A. (Ed.). (2011). Sensory marketing: Research on the sensuality of products. Routledge.

Krishna, A., Lee, S. W., Li, X. y Schwarz, N. (2017). Embodied cognition, sensory marketing, and the conceptualization of consumers' judgment and decision processes: introduction to the issue. Journal of the Association for Consumer Research, 2(4), 377-381. https://doi.org/10.1086/694453 
Marín Dueñas, P. P. y Gómez Carmona, D.

Comportamiento de compra y marketing de los sentidos: un análisis de su influencia en los compradores de moda

Kühn, S. W. y Petzer, D. J. (2018). Fostering purchase intentions toward online retailer websites in an emerging market: An SOR perspective. Journal of Internet Commerce, 17(3), 255-282. https:// doi.org/10.1080/15332861.2018.1463799

Kumar, P. (2014). Multisensory marketing: creating sustainability perspective in various sectors. Asia-Pacific Journal of Management Research and Innovation, 10(1), 8995. https:// doi.org/10.1177/2319510X14529489

Lindstrom, M. (2005). Broad sensory branding. Journal of Product $\mathcal{E}$ Brand Management. 14 (2), 84-87. https:// doi.org/10.1108/10610420510592554

Lowe, J., Maggioni, I. y Sands, S. (2018). Critical success factors of temporary retail activations: A multi-actor perspective. Journal of Retailing and Consumer Services, 40, 74-81. https:// doi.org/10.1016/j.jretconser.2017.09.005

North, A. C., Sheridan, L. P. y Areni, C. S. (2016). Music congruity effects on product memory, perception, and choice. Journal of Retailing, 92(1), 83-95. https://doi.org/10.1016/j.jretai.2015.06.001

North, A. C., Shilcock, A. y Hargreaves, D. J. (2003). The effect of musical style on restaurant customers' spending. Environment and behavior, 35(5), 712-718. https://doi.org/10.1177/0013916503254749

Manzano, R., Gavilán, D., Avello, M., Abril, C. y Serra, T. (2012). Marketing sensorial: Comunicar con los sentidos en el punto de venta. Pearson Educación.

Mehta, R. y Zhu, R. J. (2009). Blue or red? Exploring the effect of color on cognitive task performances. Science, 323(5918), 1226-1229. https://doi.org/10.1126/science.1169144

Michel, A., Baumann, C. y Gayer, L. (2017). Gracias por la música, ¿o no? Los efectos de la música en la tienda en la configuración del servicio. Journal of Retailing and Consumer Services, 36, 21-32.

Milliman, R. E. (1982). Using background music to affect the behavior of supermarket shoppers. Journal of marketing, 46(3), 86-91.

Monitor ${ }^{\mathrm{TM}}$. (2017). Getting It Right. https://lifestylemonitor.cottoninc.com/gettingit-right/.

Morgan, T. (2016). Visual merchandising: escaparates e interiores comerciales (3a. ed.). Editorial Gustavo Gili.

Morone, A., Nemore, F. y Schirone, D. A. (2018). Sales impact of servicescape's rational stimuli: A natural experiment. Journal of Retailing and Consumer Services, 45, 256-262. https://doi.org/10.1016/j.jretconser.2018.09.011 
Marín Dueñas, P. P. y Gómez Carmona, D.

Comportamiento de compra y marketing de los sentidos: un análisis de su influencia en los compradores de moda

Observatorio Cetelem (2020). Lugar preferido por los españoles para realizar sus compras en general: comercios de barrio vs. grandes cadenas de distribución de 2015 a 2020. https://es.statista.com/estadisticas/532410/comercios-de-barrio-ograndes-cadenas-preferencias-del-consumidor-espana/

Ortegón, L. y Gómez, A. G. (2016). Gestión del marketing sensorial sobre la experiencia del consumidor. Revista de Ciencias Sociales (Ve), 22(3), 67-83.

Palomares, R. (2009). Merchandising: Teoría, práctica y estrategia. ESIC

Price Waterhouse Coopers (2018). https://www.pwc.es/es/retail-consumo/2018global-consumer-insights-survey.html

Romero, M. y Biswas, D. (2016). Healthy-left, unhealthy-right: Can displaying healthy items to the left (versus right) of unhealthy items nudge healthier choices? Journal of Consumer Research, 43(1), 103-112. https://doi.org/10.1093/jcr/ucw008

Reynolds-McIlnay, R. y Morrin, M. (2019). Increasing shopper trust in retailer technological interfaces via auditory confirmation. Journal of Retailing, 95(4), 128 142. https:// doi.org/10.1016/j.jretai.2019.10.006

Rodríguez, C. L. (2018). The role of visual merchandising to position fashion retailers: a key place in Spanish Literature. aDResearch: Revista Internacional de Investigación en Comunicación, (17), 8-29. https://doi.org/10.7263/adresic-017-01

Roggeveen, A. L., Grewal, D., Townsend, C. y Krishnan, R. (2015). The impact of dynamic presentation format on consumer preferences for hedonic products and services. Journal of Marketing, 79(6), 34-49. https:// doi.org/10.1509/jm.13.0521

Statista (2020). Productos online comprados en España. https:/ / es.statista.com/estadisticas/499435/productos-online-comprados-onlineespana/

\section{AUTOR/ES:}

\section{Pedro Pablo Marín Dueñas}

Doctor en Ciencias Sociales por la Universidad de Cádiz. Profesor Ayudante Doctor en el departamento de Marketing y Comunicación, en la Facultad de Ciencias Sociales y de la Comunicación de la Universidad de Cádiz, es coordinador del Grado en Marketing e Investigación de Mercados y del doble Grado en Marketing e Investigación de Mercados y Turismo de la Universidad de Cádiz. Es miembro investigador del Instituto de Investigación Universitario para el Desarrollo Social Sostenible y Director de la Radio Universitaria INDESS Media Radio. Su línea de investigación principal es la comunicación en las organizaciones, contando con más 
Marín Dueñas, P. P. y Gómez Carmona, D.

Comportamiento de compra y marketing de los sentidos: un análisis de su influencia en los compradores de moda

de 40 publicaciones tanto en revistas de impacto (JCR, SJR, ESCI) como en editoriales indexadas en el SPI.

Orcid ID: https://orcid.org/0000-0001-8692-1174

\section{Diego Gómez-Carmona:}

Doctor en Ciencias Económicas y Empresariales por la Universidad de Granada. Profesor Sustituto Interino en el departamento de Marketing y Comunicación, en la Facultad de Ciencias Sociales y de la Comunicación de la Universidad de Cádiz. Es miembro investigador del Instituto de Investigación Universitario para el Desarrollo Social Sostenible y forma parte de la Comisión de Investigación del INDESS. Su línea de investigación principal es la neurociencia del consumidor. Sus trabajos más recientes han aparecido en revistas indexadas en JCR, como Physiology \& Behavior o DYNA y se han presentado en conferencias internacionales como AEMARK o el Congreso Hispano-Lusitano de Gestión Científica.

Orcid ID: https:// orcid.org/0000-0002-0146-5956 